\title{
A risk-based intervention approach to eliminate diabetes health disparities
}

\author{
Neil S. Calman ${ }^{1}$, Diane Hauser $^{1}$, Lilli Schussler $^{1}$ and Casey Crump ${ }^{1,2}$ \\ ${ }^{1}$ Department of Family Medicine and Community Health, Icahn School of Medicine at Mount Sinai, New York, NY, USA \\ ${ }^{2}$ Department of Population Health Science and Policy, Icahn School of Medicine at Mount Sinai, New York, NY, USA
}

\begin{abstract}
Type 2 diabetes plays a major role in racial/ethnic health disparities. We conducted the first study to examine whether multifaceted interventions targeting patients with poorly controlled diabetes $\left(\mathrm{HgbA}_{1 \mathrm{c}}>9 \%\right)$ can reduce racial/ethnic disparities in diabetes control. Among 4595 patients with diabetes at a Federally Qualified Health Center in New York, a higher percentage of blacks (32\%) and Hispanics/Latinos (32\%) had poorly controlled diabetes than whites $(25 \%)$ at baseline (prevalence ratio, $1.28 ; 95 \% \mathrm{Cl}, 1.14-1.43$; $P<0.001$ ). After four years, this percentage was reduced in all groups (blacks, $21 \%$; Hispanics/Latinos, $20 \%$; whites, $20 \% ; P<0.001$ for each relative to baseline). Disparities in diabetes control also were significantly reduced (change in disparity relative to whites: blacks, $P=0.03$; Hispanics/Latinos, $P=0.008$ ). In this diverse population, interventions targeting patients with poorly controlled diabetes not only improved diabetes control in all racial/ethnic groups, but significantly reduced disparities. This approach warrants further testing and may help reduce disparities in other populations.
\end{abstract}

Key words: delivery of health care; diabetes mellitus; healthcare disparities; health status disparities

Received 24 April 2017; revised 31 December 2017; accepted 8 January 2018;

first published online 8 February 2018

\section{Introduction}

In 2010, the US Department of Health and Human Services issued a Disparities Action Plan to achieve a 'a nation free of disparities in health and health care' (US Department of Health and Human Services, 2010). Type 2 diabetes mellitus plays a major role in health disparities because of its high prevalence and disease burden that disproportionately affect racial/ethnic minorities. In the past two decades, type 2 diabetes mellitus tripled in prevalence and now affects more than 1 in 10 US adults (Centers for Disease Control and Prevention, 2014; Geiss et al., 2014). Non-Hispanic blacks and Hispanics/Latinos have a 60-80\%

Correspondence to: Casey Crump, Departments of Family Medicine and Community Health and of Population Health Science and Policy, Icahn School of Medicine at Mount Sinai, One Gustave L. Levy Place, Box 1077, New York, NY 10029. Email: casey.crump@mssm.edu higher prevalence than non-Hispanic whites, and significantly higher rates of diabetes complications including kidney and heart disease, blindness, amputations, and premature death (Lanting et al., 2005; Spanakis and Golden, 2013). Modifiable environmental and social factors are major contributors to these disparities, including socioeconomic barriers to primary prevention and equitable health care (Walker et al., 2016). Their large population health impact is expected to increase further because of the ongoing diabetes epidemic and demographic trends for Hispanics/ Latinos and other minority groups, who are projected to account for half the US population by 2050 (Centers for Disease Control and Prevention, 2014).

Although diabetes-related disparities have been repeatedly described (Centers for Disease Control and Prevention, 2014), evidence-based knowledge about solutions remains limited. Prior studies have suggested that generalized quality improvement (QI) directed at all patients with diabetes has

(C) Cambridge University Press 2018 
largely failed to reduce disparities in diabetes control (Peek et al., 2007; Wilkes et al., 2011). We hypothesized that more intensive interventions directed at highest-risk patients will not only improve diabetes control among all racial/ethnic groups, but reduce overall disparities, because the highest-risk patients disproportionately include racial/ethnic minorities. We sought to test this hypothesis in a highly diverse patient population in a large network of community health centers. To our knowledge, this approach has not previously been tested as a strategy for reducing racial/ethnic disparities in diabetes outcomes.

\section{Methods}

We conducted a cohort study in a large network of Federally Qualified Health Centers in New York State. The Institute for Family Health (IFH), affiliated with the Icahn School of Medicine at Mount Sinai, provides comprehensive primary care to a historically underserved population of $\sim 100000$ patients in New York City and the Hudson Valley. In 2006-2008, IFH implemented an intensive intervention program directed at patients with poorly controlled diabetes (latest hemoglobin $\left.\mathrm{A} 1 \mathrm{c}\left[\mathrm{HbA}_{1 \mathrm{c}}\right]>9 \%\right)$. The interventions were multifaceted and included a systemwide electronic health record (EHR)-based registry, diabetes care managers, certified diabetes educator visits, group visits in English and Spanish to promote patient education and peer support, special outreach to patients with overdue or missed clinic appointments, EHR-based clinical decision supports for diabetes care standards, and on-site $\mathrm{HbA}_{1 \mathrm{c}}$ testing to enable more rapid treatment changes when warranted. Implementation of these interventions has been described previously in more detail in the context of transitioning to a Patient Centered Medical Home (Calman et al., 2013).

We examined the effects of these interventions on racial/ethnic disparities in diabetes control among all adult patients (age $>18$ years) with a diagnosis of diabetes mellitus at baseline (20062008) and at least one $\mathrm{HbA}_{1 \mathrm{c}}$ measurement each year during the follow-up period (2008-2012; $n=4595)$. Diabetes mellitus was identified based on International Classification of Diseases (ICD) codes in the EHR (ICD-9, 250; ICD-10, E11-E14).
We used Poisson regression with robust standard errors to determine prevalence ratios and 95\% confidence intervals for poorly controlled diabetes $\left(\mathrm{HbA}_{1 \mathrm{c}}>9 \%\right)$ among non-Hispanic blacks or Hispanics/Latinos relative to non-Hispanic whites at baseline and after four years of follow-up. We performed difference-in-differences analyses to examine the change in disparity in the prevalence of poorly controlled diabetes among non-Hispanic blacks or Hispanics/Latinos versus non-Hispanic whites at the end of follow-up relative to baseline. All analyses were adjusted for age, sex, and type of health insurance (Medicaid, Medicare, private insurance, or uninsured).

In secondary analyses, we examined potential interactions between race/ethnicity and age, sex, or type of health insurance in relation to poorly controlled diabetes or the change in disparities in this outcome, using likelihood ratio tests. All statistical tests were two-sided and used an $\alpha$-level of 0.05. All analyses were conducted using Stata version 14.1. This study was approved by the Institutional Review Board of the Institute for Family Health (\#2241).

\section{Results}

In this cohort of 4595 patients with diabetes, $37.0 \%$ were Hispanic/Latino, 27.9\% were non-Hispanic black, $23.9 \%$ were non-Hispanic white, $7.3 \%$ were other races, and $3.9 \%$ had missing race and ethnicity information. We focused on the three largest racial/ ethnic groups (Hispanics/Latinos, non-Hispanic blacks, and non-Hispanic whites) because other groups had insufficient numbers for well-powered analyses. Nearly half $(45.8 \%)$ of this cohort was enrolled in Medicaid. Other patient characteristics are shown in Table 1.

During four years of follow-up, the prevalence of poorly controlled diabetes $\left(\mathrm{HbA}_{1 \mathrm{c}}>9 \%\right)$ not only declined in each of these racial/ethnic groups, but racial/ethnic disparities in this outcome were substantially reduced (Figure 1). At baseline, a higher percentage of either non-Hispanic blacks $(32 \%)$ or Hispanics/Latinos (32\%) had poorly controlled diabetes than non-Hispanic whites $(25 \%)$ (prevalence ratio for each, $1.28 ; 95 \% \mathrm{CI}, 1.14-1.43 ; P<0.001)$. After 4 years, this percentage was significantly reduced in all groups (non-Hispanic blacks, 21\%; Hispanics/Latinos, 20\%; non-Hispanic whites, 20\%; 
Table 1 Characteristics of patients with diabetes, 2008$2012(n=4595)$

\begin{tabular}{lr}
\hline & $n(\%)$ \\
\hline Age (years) & \\
$<40$ & $524(11.4)$ \\
$40-64$ & $3584(78.0)$ \\
$\geqslant 65$ & $487(10.6)$ \\
Sex & \\
Female & $2655(57.8)$ \\
Male & $1490(42.2)$ \\
Race or ethnicity & \\
Non-Hispanic black & $1282(27.9)$ \\
Hispanic/Latino & $1700(37.0)$ \\
Non-Hispanic white & $1098(23.9)$ \\
Asian & $106(2.3)$ \\
American Indian & $18(0.4)$ \\
Other & $214(4.7)$ \\
Missing & $177(3.9)$ \\
Insurance type & \\
Medicaid & $2105(45.8)$ \\
Medicare & $515(11.2)$ \\
Private & $1336(29.1)$ \\
Uninsured & $639(13.9)$ \\
\hline
\end{tabular}

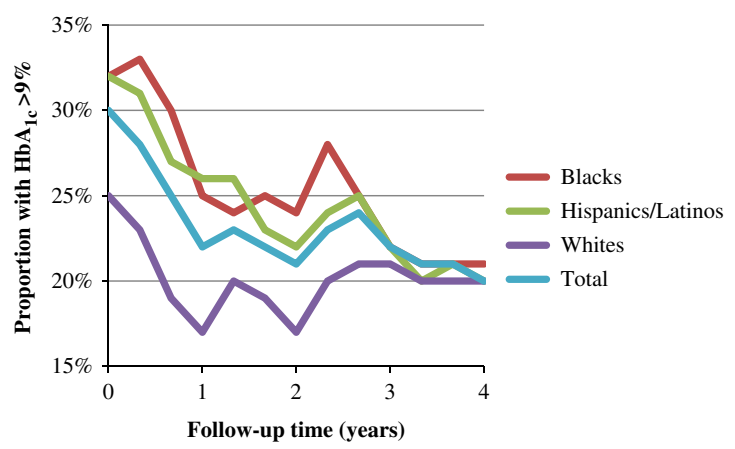

Figure 1 Percentage of patients with diabetes who had $\mathrm{HbA}_{1 \mathrm{c}}>9 \%$ during 2008-2012, stratified by race/ethnicity.

$P<0.001$ for each relative to baseline). In addition, racial/ethnic disparities in the prevalence of poorly controlled diabetes were significantly reduced or eliminated (change in disparity relative to nonHispanic whites: non-Hispanic blacks, $P=0.03$; Hispanics/Latinos, $P=0.008$ ). Figure 1 shows the change in this outcome across the study period, stratified by race/ethnicity.

In secondary analyses, we found no significant interactions between race/ethnicity and age, sex, or health insurance type in relation to poorly controlled diabetes or disparities in this outcome
$(P>0.05$ for each interaction). Among patients who were not included in the analyses because of insufficient follow-up $(n=1228), \mathrm{HbA}_{1 \mathrm{c}}$ level did not vary significantly among racial/ethnic groups $(P=0.27$, Kruskal-Wallis test $)$, thus there was no evidence that $\mathrm{HbA}_{1 \mathrm{c}}$ differences among these patients accounted for the observed reduction in disparities.

\section{Discussion}

To our knowledge, this is the first study to test whether more intensive clinical interventions directed at patients with poorly controlled diabetes can effectively reduce racial/ethnic disparities. In a highly diverse population, we found that this approach not only improved diabetes control in all racial/ethnic groups, but eliminated racial/ethnic disparities in this outcome. Additional follow-up will be needed to examine long-term sustainability of these effects and whether disparities in other outcomes including diabetes complications are also reduced.

Most studies of interventions to address diabetesrelated disparities have focused on generalized QI in diabetes care, under the premise that 'a rising tide floats all boats' (Peek et al., 2007). Such studies have frequently reported overall improvements in diabetes care processes (eg, $\mathrm{HbA}_{1 \mathrm{c}}$ test frequency) but mixed results for diabetes control ( $\mathrm{HbA}_{1 \mathrm{c}}$ levels), and have seldom examined other diabetes outcomes (Peek et al., 2007; Wilkes et al., 2011). Remarkably, few studies have included a comparison group of non-Hispanic whites to enable direct testing of changes in racial/ethnic disparities, and the few that have done so have found that QI interventions had no effect on disparities in diabetes control. Even when diabetes control improved among different racial/ethnic groups, racial/ethnic disparities persisted unchanged (Peek et al., 2007; Sequist et al., 2006).

Although QI interventions play an important role in improving overall care, new strategies are clearly needed for reducing diabetes-related disparities. The present study examined more intensive interventions directed at patients with the poorest prognosis based on disease control. This 'risk-based affirmative health care action' approach essentially weights the allocation of clinical resources toward any racial/ethnic groups 
that are disproportionately represented among the highest-risk patients. This strategy has the potential not only to improve diabetes outcomes among all patients irrespective of race/ethnicity, but simultaneously to reduce racial/ethnic disparities.

The present study has several strengths, including a racially diverse cohort of patients with diabetes that was sufficiently large to examine disparities with high statistical power. Our analyses included not only racial/ethnic minorities but non-Hispanic whites as a comparison group to enable direct testing of changes in disparities in diabetes control. We examined a multifaceted intervention program that targeted patients, providers, and the health delivery system, which is likely to be more effective than those targeting only one of these domains (Peek et al., 2007).

Certain limitations should also be acknowledged. First, this study examined a multifaceted intervention program as a whole, rather than the effects of different interventions separately. Mediation analyses in future studies may be useful to delineate which intervention components are most effective at reducing racial/ethnic disparities. The present study also focused on only one outcome, racial/ethnic disparities in $\mathrm{HbA}_{1 \mathrm{c}}$ control, which was assessed during four years of follow-up. Longer follow-up will be needed to examine effects on disparities in other diabetes outcomes that take longer to manifest, such as cardiovascular and renal complications. In addition, this study included a historically underserved patient population that has a high overall prevalence and severity of diabetes and comorbidities. Additional studies will be needed to replicate this approach and assess its generalizability in other health-care settings and populations.

The immense societal costs of health disparities are well-documented, including higher health care expenditures, total disease burden, and premature mortality (US Department of Health and Human Services, 2010). Reducing disparities is important not only for social justice, but would benefit everyone by enhancing the overall quality of health-care delivery, lowering costs, and improving population health (US Department of Health and Human Services, 2010). Prior efforts to reduce diabetes-related disparities through generalized QI have had limited success. In a highly diverse, historically underserved population, we found for the first time that a risk-based intervention strategy effectively reduced racial/ethnic disparities in diabetes control. This approach warrants further replication and testing in other settings, which may help advance our knowledge beyond describing disparities toward developing more effective and scalable solutions.

\section{Conflicts of Interest}

None.

\section{Ethical Standards}

The authors assert that all procedures contributing to this work comply with the ethical standards of the relevant national and institutional guidelines on human experimentation and with the Helsinki Declaration of 1975, as revised in 2008.

\section{References}

Calman, N.S., Hauser, D., Weiss, L., Waltermaurer, E., Molina-Ortiz, E., Chantarat, T. and Bozack, A. 2013: Becoming a patient-centered medical home: a 9-year transition for a network of Federally Qualified Health Centers. Annals of Family Medicine 11 (Suppl 1), S68-S73.

Centers for Disease Control and Prevention. 2014: National Diabetes Statistics Report: Estimates of Diabetes and Its Burden in the United State. Atlanta, GA.

Geiss, L.S., Wang, J., Cheng, Y.J., Thompson, T.J., Barker, L., Li, Y., Albright, A.L. and Gregg, E.W. 2014: Prevalence and incidence trends for diagnosed diabetes among adults aged 20 to 79 years, United States, 1980-2012. Journal of the American Medical Association 312, 1218-1226.

Lanting, L.C., Joung, I.M., Mackenbach, J.P., Lamberts, S.W. and Bootsma, A.H. 2005: Ethnic differences in mortality, end-stage complications, and quality of care among diabetic patients: a review. Diabetes Care 28, 2280-288.

Peek, M.E., Cargill, A. and Huang, E.S. 2007: Diabetes health disparities: a systematic review of health care interventions. Medical Care Research and Review 64 (Suppl 5), 101S-56S.

Sequist, T.D., Adams, A., Zhang, F., Ross-Degnan, D. and Ayanian, J.Z. 2006: Effect of quality improvement on racial disparities in diabetes care. Archives of Internal Medicine 166, 675-81.

Spanakis, E.K. and Golden, S.H. 2013: Race/ethnic difference in diabetes and diabetic complications. Current Diabetes Reports 13, 814-23. 
US Department of Health and Human Services. 2010: HHS action plan to reduce racial and ethnic health disparities: a nation free of disparities in health and health care. Washington, DC: US Department of Health and Human Services.

Walker, R.J., Strom Williams, J. and Egede, L.E. 2016: Influence of race, ethnicity and social determinants of health on diabetes outcomes. American Journal of Medical Science 351, 366-73.

Wilkes, A.E., Bordenave, K., Vinci, L. and Peek, M.E. 2011: Addressing diabetes racial and ethnic disparities: lessons learned from quality improvement collaboratives. Diabetes Management (London) 1, 653-60. 